\title{
Suspended low-loss germanium waveguides for the longwave-infrared
}

\author{
A. Osman ${ }^{1,}$, M. Nedeljkovic ${ }^{1}$, J. Soler Penades ${ }^{1}$, Y. Wu ${ }^{1}$, Z. Qu ${ }^{1}$, A. Z. KhokhaR ${ }^{1}$, K. \\ Debnath $^{1,2}$, And G. Z. Mashanovich ${ }^{1}$ \\ ${ }^{1}$ Optoelectronics Research Centre, University of Southampton, Southampton SO17 1BJ, UK \\ ${ }^{2}$ Indian Institute of technology Kharagpur Electronics \& Electrical Communication Engineering Department, IIT Kharagpur, Kharagpur, WB 721302 \\ *Corresponding author: amo1g15@soton.ac.uk
}

Compiled November 5, 2018

Germanium is a material of high interest for midinfrared (MIR) integrated photonics due to its CMOS compatibility and its wide transparency window covering the 2-15 $\mu \mathrm{m}$ spectral region exceeding the 4 and 8 $\mu \mathrm{m}$ limit of the Silicon-on-Insulator (SOI) platform and Si material respectively. In this Letter, we report suspended germanium waveguides operating at a wavelength of $7.67 \mu \mathrm{m}$ with a propagation loss of $2.6 \pm 0.3$ $\mathrm{dB} / \mathrm{cm}$. To our knowledge, this is the first demonstration of low-loss suspended germanium waveguides at such a long wavelength. Suspension of the waveguide is achieved by defining holes alongside the core providing access to the buried oxide layer and the underlying Si layer so that they can be wet etched using HF and TMAH respectively. Our MIR waveguides create a new path towards long wavelength sensing in the fingerprint region. () 2018 Optical Society of America

OCIS codes: (130.0130) Integrated optics; (130.5990) Semiconductors; (230.7370) Waveguides.

\section{http://dx.doi.org/10.1364/ao.XX.XXXXXX}

The mid-infrared (MIR) spectral region is attractive for the development of various devices for gas, chemical and biological sensing [1] as well as for spectroscopic applications [2]. That is due to the fact that vibrational transition energies of numerous molecules fall in the wavelength range of 3-20 $\mu \mathrm{m}$. Molecular absorption spectra in this range contain intense and distinctive features, providing a unique 'molecular fingerprint'. Some of these substances require the use of wavelengths above $7 \mu \mathrm{m}$ [3]. For example, sulfur dioxide, which is produced as a byproduct of the burning of fossil fuels contaminated with sulfur compounds, has strong absorption near $7.6 \mu \mathrm{m}$ [4]. In addition, volatile organic compounds (VOCs) such as monochlorobenzene (MCB) and chloroform (CF), are contaminants in drinking water and have absorption peaks near $13 \mu \mathrm{m}$ [5]. Therefore, there is an apparent need for the development of photonic integrated circuits that can operate throughout the MIR. Furthermore, the atmospheric transmission windows in the 3-5 $\mu \mathrm{m}$ and 8-13 $\mu \mathrm{m}$ ranges can extend IR technologies to longer distances for appli- cations such as remote explosive detection [6], thermal imaging [7], and free-space communications [8].

Silicon, which has been widely used for photonic integrated circuits in the near-IR (NIR), has recently been used for various developments in the MIR spectral range as well. Numerous MIR photonic integrated devices have been demonstrated utilising silicon-based platforms. In NIR Si photonics, SOI $[9,10]$ has been the dominant platform because SOI wafers are easily available and the fabrication techniques are mature and well-developed. However, due to the high absorption of $\mathrm{SiO}_{2}$ above $4 \mu \mathrm{m}$ (i.e., $\sim 450 \mathrm{~dB} / \mathrm{cm}$ at $5 \mu \mathrm{m}$, rising to $\sim 2.5 \times 10^{4} \mathrm{~dB} / \mathrm{cm}$ at $7.6 \mu \mathrm{m}$ ) [11], $\mathrm{SOI}$ is unsuitable for MIR applications at wavelengths longer than $4 \mu \mathrm{m}$.

Therefore, there is an apparent need for the investigation of alternative platforms to SOI in order to enable the development of devices which will operate at $>4 \mu \mathrm{m}$. A different approach used in recent developments to overcome the optical mode overlap with $\mathrm{SiO}_{2}$ is to suspend the $\mathrm{Si}$ waveguide core. In [12-14] two dry-etch steps were carried out to initially define the $\mathrm{Si}$ (or $\mathrm{Ge}$ ) rib waveguide, and then to create an array of holes alongside the waveguide that allow the local removal of the buried oxide (BOX) by wet etching in Hydrofluoric acid (HF). In [15-17], an alternative approach was presented that consists of a strip waveguide with subwavelength grating (SWG) holes that acted as a cladding for the lateral confinement of the optical mode as well as access to the BOX. By utilising this approach, a minimum loss of $0.82 \mathrm{~dB} / \mathrm{cm}$ was achieved at $3.8 \mu \mathrm{m}[15]$ and $3.1 \mathrm{~dB} / \mathrm{cm}$ at $7.67 \mu \mathrm{m}$ [17]. The same approach has recently been implemented to create suspended Si slot waveguides [18] at $2.25 \mu \mathrm{m}$. However, the intrinsic absorption of silicon at wavelengths longer than $7.7 \mu \mathrm{m}$ (i.e. $\sim 2.1 \mathrm{~dB} / \mathrm{cm}$ at $7.67 \mu \mathrm{m}$, increasing to $4.6 \mathrm{~dB} / \mathrm{cm}$ at $9 \mu \mathrm{m}$ and $9.3 \mathrm{~dB} / \mathrm{cm}$ at $11 \mu \mathrm{m}$ [19]) renders silicon-based MIR integrated devices unable to operate in the long-wave infrared fingerprint spectral region $(8-15 \mu \mathrm{m})$.

Germanium has become a material of high interest for MIR integrated photonics due to its CMOS compatibility, wide spectral transparency window $(2-15 \mu \mathrm{m})$ [20], high refractive in$\operatorname{dex}(n \sim 4$ at $7.67 \mu \mathrm{m})$ and high third-order nonlinear susceptibility $\left(\sim 10^{-18} \mathrm{~m}^{2} / \mathrm{V}^{2}\right)$ [20]. These features enable the exploitation of a wider wavelength range in the MIR, as well as the development of small-footprint and high efficiency nonlinear devices. Since 2012 several photonic devices for the MIR 
have been demonstrated using germanium-based platforms, including Germanium-on-Silicon (GOS) waveguides, demonstrating a propagation loss of $2.5 \mathrm{~dB} / \mathrm{cm}$ at $7.58 \mu \mathrm{m}$ [21] and less than $10 \mathrm{~dB} / \mathrm{cm}$ at wavelengths as long as $11.25 \mu \mathrm{m}$ [22], and other passive devices [23], germanium-on-SOI waveguides [24], germanium-on- $\mathrm{Si}_{3} \mathrm{~N}_{4}$ waveguides [25], germanium-on-insulator (GOI) waveguides [26, 27], SiGe/Si waveguides [28-30] and suspended germanium devices $[13,14,31,32]$ at wavelengths up to $3.8 \mu \mathrm{m}$.

Using suspended Ge has the principal advantage of avoiding material losses from any claddings so that Ge's entire transparency range can be exploited. A further advantage of suspended Ge compared to the GOS platform is that it has a high and symmetrical refractive index contrast between the core and upper and lower air claddings. In the case of GOS, there is a high index contrast between the Ge core $(\Delta n \approx 3.0$ at $7.67 \mu \mathrm{m})$ and upper air cladding, but low contrast with the Si lower cladding $(\Delta n \approx 0.6$ at $7.67 \mu \mathrm{m})$. This results in the need for thicker rib waveguides which in turn will result in a weak mode overlap of the evanescent field with the upper air cladding. Therefore, suspended Ge enables the development of thinner waveguides that exhibit larger evanescent field. This is a great advantage for sensing applications, in terms of sensitivity, where high mode overlap with an analyte surrounding the waveguide is desirable.

To our knowledge, suspended Ge waveguides have not been demonstrated at wavelengths beyond $3.8 \mu \mathrm{m}$. In this letter we report suspended Ge waveguides with a propagation loss of $2.6 \mathrm{~dB} / \mathrm{cm}$ at $\lambda=7.67 \mu \mathrm{m}$. Our suspended Ge platform is expected to enable diverse on-chip applications in sensing and spectroscopy over a wide MIR spectral range, which in turn will boost the development of MIR germanium photonics.

A schematic representation of the demonstrated suspended waveguide is illustrated in Fig. 1.

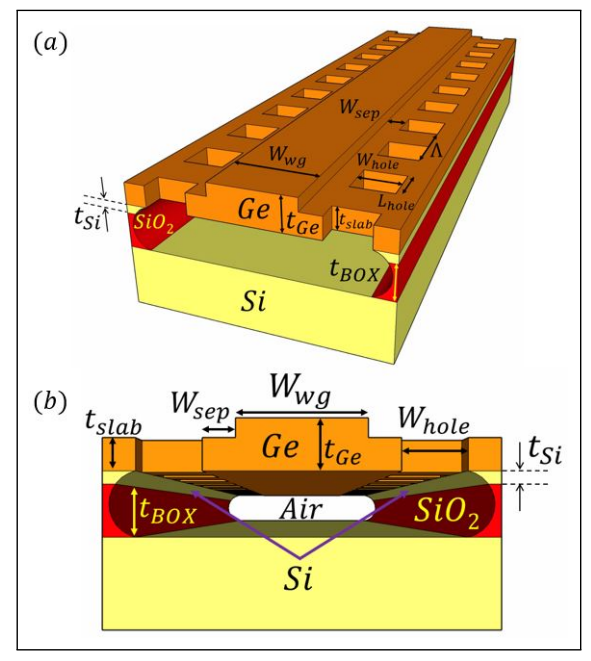

Fig. 1. 3D schematic ((a) overview and (b) front view) of the proposed suspended germanium waveguide.

The guiding structure is a rib waveguide, based on a Geon-SOI platform. The wafers were fabricated using Ge-on-SOI with a $1 \mu \mathrm{m}$ Ge layer grown by RPCVD on SOI in which the Si layer was thinned from $220 \mathrm{~nm}$ down to $60 \mathrm{~nm}$. This platform was chosen over the GOI due to the fact that current methods for fabricating GOI films require tight process control and limit the range of thicknesses that can be obtained [33]. Moreover, bonded GOI wafers are not commercially available. The Ge layer of thickness $t_{G e}$ was grown on SOI which comprises a Si layer and a BOX of thickness $t_{S i}$ and $t_{B O X}$ respectively. Waveguide modelling was carried out using the FDE solver in the Lumerical Mode Solutions commercial software package (Fig. 2).

Rib waveguides were designed for single TE mode propagation at $\lambda=7.67 \mu \mathrm{m}$, with waveguide thickness $t_{G e}$, width $W_{w g g}$, and etch depth $t_{G e}-t_{\text {slab }}$ (Fig. 1). The slab thickness was selected such that mechanical stability is ensured and yet the mode is well-confined when propagating through bends. For bend radii larger than $200 \mu \mathrm{m}$ the simulated bend loss was $\leq 0.1$ $\mathrm{dB} / 90^{\circ}$ bend. The holes providing access to the BOX were designed to have sufficient distance from the waveguide core such that the fraction of the optical power propagating through them does not exceed $0.1 \%$. The dimensions are: hole width $W_{\text {hole }}$, hole length $L_{\text {hole }}$, period $\Lambda$ and separation width $W_{\text {sep }}$ (Fig. 1). The final selected values of the whole structure are indicated in Table 1. Fig. 2 shows the cross-section of the fundamental TE mode corresponding to the dimensions in Table 1 . The simulated optical mode confinement of the fundamental TE mode at 7.67 $\mu \mathrm{m}$ in air, using these dimensions, is $\sim 15 \%$ as opposed to $\sim 2.5 \%$ in GOS in the waveguides presented in [21].

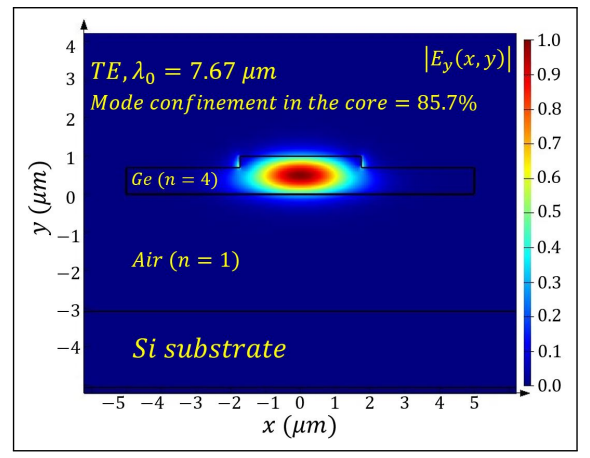

Fig. 2. Cross section of the simulated fundamental TE mode at $\lambda=7.67 \mu \mathrm{m}$.

At such long wavelengths, the loss due to the substrate leakage becomes a limiting factor. Therefore, having a sufficient air gap thickness between the waveguide and the substrate is crucial for operation at longer wavelengths. Fig. 3 shows the loss of $1 \mu \mathrm{m}$ thick Ge, due to leakage to the substrate as a function of the air gap thickness. The graph was created by sweeping the position of the lower $z$ boundary in the FDE solver, upon setting it to perfectly matched layer (PML) and obtaining the corresponding loss values. It is apparent that at wavelengths longer than $12 \mu \mathrm{m}$ the $3 \mu \mathrm{m}$ air gap becomes insufficient for the mode confinement in the waveguide.

Table 1. Designed dimensions of the suspended Ge waveguides at $\lambda_{0}=7.67 \mu \mathrm{m}$

\begin{tabular}{cc|cc}
\hline$W_{w g}$ & $3.5 \mu \mathrm{m}$ & $W_{\text {sep }}$ & $3 \mu \mathrm{m}$ \\
$W_{\text {hole }}=L_{\text {hole }}$ & $1 \mu \mathrm{m}$ & $\Lambda$ & $3 \mu \mathrm{m}$ \\
$t_{\text {Ge }}$ & $1 \mu \mathrm{m}$ & $t_{\text {Slab }}$ & $700 \mathrm{~nm}$ \\
$t_{S i}$ & $60 \mathrm{~nm}$ & $t_{B O X}$ & $3 \mu \mathrm{m}$ \\
\hline
\end{tabular}

Light coupling was achieved using non-suspended 2D grating couplers that were designed to have an etch depth of $t_{g r}=300 \mathrm{~nm}$, a period in the x-axis of $\Lambda_{g r, x}=2.63 \mu \mathrm{m}$ with a duty cycle of $D C_{x}=0.5$, a period in the y-axis of $\Lambda_{g r, y}=1 \mu \mathrm{m}$ with a duty cycle of $D C_{y}=0.7$ and to be $115 \mu \mathrm{m}$ long and 20 
$\mu \mathrm{m}$ wide. The gratings were designed to be $2-\mathrm{D}$ and partially etched to minimize back reflections due to the high refractive index contrast; hence, suspending them could only be achieved via holes alongside the $20 \mu \mathrm{m}$ wide grating region. That would dramatically increase the wet etching time, compromising the mechanical stability of the waveguides. In addition, in this initial waveguide demonstration, high excess loss from $\mathrm{SiO}_{2}$ absorption in the grating coupler and access waveguide region was not a major concern.

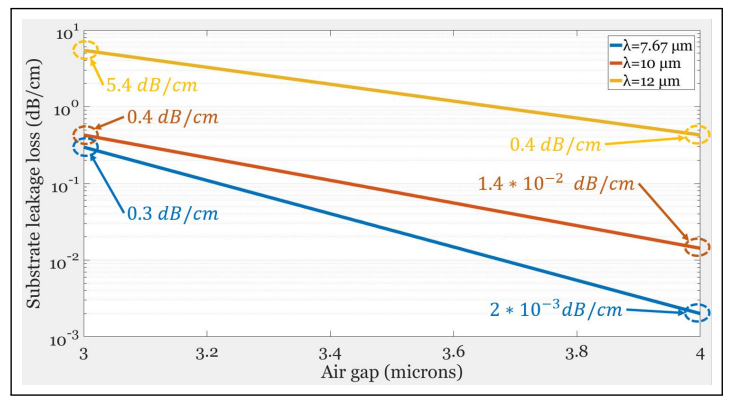

Fig. 3. Substrate leakage loss as a function of the air gap for the waveguide dimensions in Table 1.

The starting point of the fabrication process illustrated in Fig. 4 is to deposit a thin layer $(100 \mathrm{~nm})$ of $\mathrm{SiO}_{2}$ using PECVD. This prevents the formation of $\mathrm{GeO}_{2}$ by the $\mathrm{O}_{2}$ plasma during stripping of the photoresist using the plasma asher. This is due to the fact that $\mathrm{GeO}_{2}$ is soluble in water with a solubility of $4.47 \mathrm{~g} / \mathrm{L}$ at $25^{\circ} \mathrm{C}$ and $10.7 \mathrm{~g} / \mathrm{L}$ at $100{ }^{\circ} \mathrm{C}$ [34]. This could cause the creation of defects during any wet process containing water including diluted HF, result in high propagation losses due to surface roughness. Once the protective layer of $\mathrm{SiO}_{2}$ is deposited, we spin-coat the sample with ZEP-520A resist and pattern it using e-beam lithography. The first lithography step defines the waveguides and the grating couplers. Then the sample is developed using ZED-N50 developer. After exposing, the following step is etching the exposed $\mathrm{SiO}_{2}$ and $\mathrm{Ge}$ using ICP (Fig. 4(b)). After stripping the remaining resist using a plasma asher, another thin layer of $\mathrm{SiO}_{2}$ was deposited and the second e-beam lithography step was carried out to define the holes. A second ICP etching step was carried out to etch the exposed $\mathrm{SiO}_{2}$ and the remaining Ge slab as well as the Si layer down to the BOX (Fig. 4(c)). After removing the remaining resist using an $\mathrm{O}_{2}$ plasma asher, the sample underwent two wet etch steps. First, the sample was immersed in 1:7 isotropic HF for 80 minutes, which caused the local removal of the BOX. The etch rate was approximately $88 \mathrm{~nm} /$ minute. Then the sample was immersed in a $25 \%$ aqueous solution of anisotropic Tetramethylammonium hydroxide (TMAH) at room temperature for 60 minutes which resulted in the complete removal of the Si layer as well as part of the Si substrate increasing the air gap below the waveguide (Fig. 4(d)). The etch rate of TMAH was approximately $7.3 \mathrm{~nm} /$ minute in the [100] crystal direction. The ratio of etch rates of Si and Ge is 1000:1. Due to the $4.2 \%$ lattice mismatch between Ge and $\mathrm{Si}$, Ge epitaxial layers on Si substrates have typical Threading Dislocation Density (TDD) in the order of $10^{6}-10^{7} \mathrm{~cm}^{-2}$ with the defects primarily being confined to the Ge-Si interface. This results in less mode interaction with the lower Ge interface reducing the propagation loss.

Fig. 5 shows scanning electron microscope (SEM) images of a fabricated device. Fig. 5(a) shows a cross section of the suspended Ge waveguide with the Si layer and BOX locally

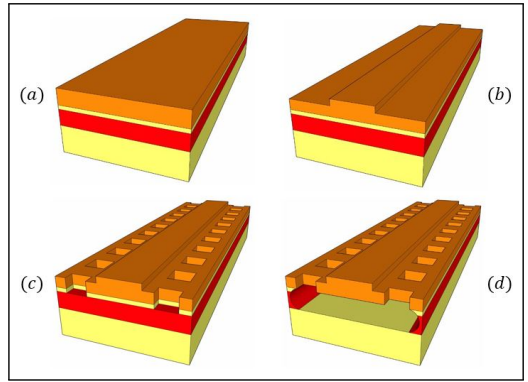

Fig. 4. Fabrication process flow of the suspended Ge waveguides. (a) Initial Ge-on-SOI platform, (b) waveguide definition, (c) holes patterning and exposure of the BOX, (d) wet etching using HF and TMAH.

removed. From Fig. 5(b) it can be seen that along with the Si layer, $440 \mathrm{~nm}$ of the substrate has also been etched increasing the air gap. This method provides the ability to precisely control the air gap enabling operation at longer wavelengths with reduced substrate leakage losses. Fig. 5(c) shows a fabricated non-suspended grating coupler and Fig. 5(d) shows a top view of the suspended Ge rib waveguide with holes alongside it.

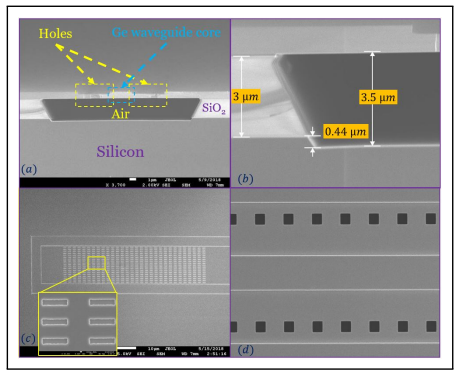

Fig. 5. SEM images of the fabricated suspended Ge waveguides. (a) Cleaved suspended Ge waveguide facet showing the complete local removal of the BOX and Si layers, (b) zoomedin image on the edges of the wet etched areas; it can be seen that the Si substrate has been etched by $440 \mathrm{~nm}$, (c) top view of a 2D grating coupler (non-suspended), (d) top view of a rib waveguide with holes alongside it.

The experimental setup utilized to characterize the fabricated waveguides comprises a single-mode continuous wave distributed-feedback quantum cascade laser (Thorlabs QD7500CM1) capable of emitting light with a maximum output power of $106 \mathrm{~mW}$ at a $\lambda=7.67 \mu \mathrm{m}$. The beam is then collimated using a black diamond-2 lens with a focal length of 1.9 $\mathrm{mm}$, modulated using a chopper wheel and then coupled into a single-mode $\mathrm{As}_{2} \mathrm{Se}_{3}$ fiber (Coractive IRT-SE-28/170) using another black diamond-2 lens with a focal length of $6.0 \mathrm{~mm}$. Light coupling into and out of the waveguides was achieved using grating couplers [Fig. 5(c)]. Light from the output grating coupler was collected via another single-mode fiber and coupled to a liquid-nitrogen-cooled $\mathrm{HgCdTe}$ detector (Infrared Associates Inc. MCT-13-1.00). The signal from the detector was amplified using a pre-amplifier before being guided to a lock-in amplifier to improve the signal-to-noise ratio.

The waveguide propagation loss was measured with the effective "cut-back" method. Fig. 6 shows the transmissions of waveguides of different lengths. It shows a propagation loss of $2.6 \pm 0.3 \mathrm{~dB} / \mathrm{cm}$. Material losses of $\mathrm{Ge}$ are negligible at this 
wavelength [35]. In addition, according to simulations, leakage losses are $0.3 \mathrm{~dB} / \mathrm{cm}$ for an air gap of $3 \mu \mathrm{m}$, decreasing to $2.4 \times$ $10^{-2}$ for an air gap of $3.5 \mu \mathrm{m}$. Therefore the propagation loss is likely caused by scattering at the waveguide side-walls and by defects due to threading dislocations in the Ge at the Ge-Si interface emanating from the $4.2 \%$ lattice mismatch between $\mathrm{Ge}$ and Si. Using the model for the calculation of the scattering loss due to side-wall roughness in [36], the standard deviation of the roughness $(\sigma)$ corresponding to the propagation loss measured in this work for a correlation length of $\mathrm{L}_{c}=50 \mathrm{~nm}$ would be $\sigma=15 \mathrm{~nm}$. The source of propagation loss cannot be accurately defined as no AFM measurement of the sidewall roughness was taken. However, we believe that a sidewall roughness of $15 \mathrm{~nm}$ is very unlikely in our processing. A typical sidewall roughness of $\sigma=5 \mathrm{~nm}$ would result in a propagation loss of $0.3 \mathrm{~dB} / \mathrm{cm}$. The rest $(2.3 \mathrm{~dB} / \mathrm{cm})$ would then emanate mostly from the threading dislocations.

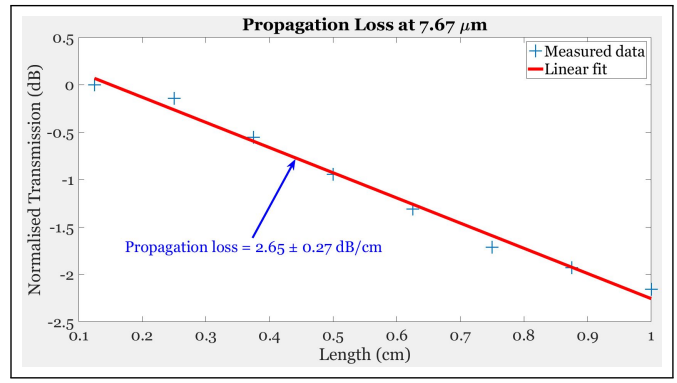

Fig. 6. Cut-back loss experimental results at $7.67 \mu \mathrm{m}$ wavelength for suspended Ge waveguides.

We have designed, fabricated, and characterized suspended germanium waveguides at a wavelength of $7.67 \mu \mathrm{m}$. The propagation loss for the waveguides was $2.6 \pm 0.3 \mathrm{~dB} / \mathrm{cm}$. The evanescent field is $\sim 15 \%$. These results show that suspended germanium waveguides fabricated using Ge-on-SOI wafers have the potential to be used throughout the MIR transparency window of germanium and for sensing applications above $8 \mu \mathrm{m}$.

\section{FUNDING INFORMATION}

Engineering and Physical Sciences Research Council (EPSRC) (EP/L01162X/1, EP/L021129/1, EP/N00762X/1). Royal Academy of Engineering (RAEng) (RF201617/16/33). Data supporting this paper are openly available from the University of Southampton repository at https://doi.org/10.5258/SOTON/D0602

\section{REFERENCES}

1. M. Sieger and B. Mizaikoff, Anal. Chem. 88, 5562 (2016). PMID: 27081763.

2. M. Sieger, J. Haas, M. Jetter, P. Michler, M. Godejohann, and B. Mizaikoff, Anal. chemistry 88, 2558 (2016).

3. R. A. Soref, S. J. Emelett, and W. R. Buchwald, J. Opt. A: Pure Appl. Opt. 8, 840 (2006).

4. NIST, "Chemistry webbook, sulfur dioxide," .

5. R. Lu, W.-W. Li, B. Mizaikoff, A. Katzir, Y. Raichlin, G.-P. Sheng, and H.-Q. Yu, Nat. protocols 11, 377 (2016).

6. C. Bauer, A. Sharma, U. Willer, J. Burgmeier, B. Braunschweig, W. Schade, S. Blaser, L. Hvozdara, A. Müller, and G. Holl, Appl. Phys. B 92, 327 (2008).

7. R. Rehm, M. Walther, J. Schmitz, J. Fleissner, J. Ziegler, W. Cabanski, and R. Breiter, Electron. Lett. 42, 577 (2006).
8. R. Martini, C. Bethea, F. Capasso, C. Gmachl, R. Paiella, E. A. Whittaker, H. Y. Hwang, D. L. Sivco, J. N. Baillargeon, and A. Y. Cho, Electron. Lett. 38, 181 (2002).

9. G. Z. Mashanovich, M. M. Milošević, M. Nedeljkovic, N. Owens, B. Xiong, E. J. Teo, and Y. Hu, Opt. Express 19, 7112 (2011).

10. M. Nedeljkovic, A. V. Velasco, A. Z. Khokhar, A. Delâge, P. Cheben, and G. Z. Mashanovich, IEEE Photonics Technol. Lett. 28, 528 (2016).

11. R. Kitamura, L. Pilon, and M. Jonasz, Appl. Opt. 46, 8118 (2007).

12. Z. Cheng, X. Chen, C. Y. Wong, K. Xu, and H. K. Tsang, IEEE Photonics J. 4, 1510 (2012).

13. T.-H. Xiao, Z. Zhao, W. Zhou, M. Takenaka, H. K. Tsang, Z. Cheng, and K. Goda, Opt. Lett. 42, 2882 (2017).

14. T.-H. Xiao, Z. Zhao, W. Zhou, C.-Y. Chang, S. Y. Set, M. Takenaka, H. K. Tsang, Z. Cheng, and K. Goda, Opt. Lett. 43, 2885 (2018).

15. J. S. Penadés, A. Ortega-Monux, M. Nedeljkovic, J. G. WangüemertPérez, R. Halir, A. Z. Khokhar, C. Alonso-Ramos, Z. Qu, I. MolinaFernández, P. Cheben, and G. Z. Mashanovich, Opt. Express 24, 22908 (2016).

16. J. S. Penadés, C. Alonso-Ramos, A. Z. Khokhar, M. Nedeljkovic, L. A. Boodhoo, A. Ortega-Monux, I. Molina-Fernández, P. Cheben, and G. Z. Mashanovich, Opt. Lett. 39, 5661 (2014).

17. J. S. Penadés, A. Sánchez-Postigo, M. Nedeljkovic, A. Ortega-Monux, J. G. Wangüemert-Pérez, Y. Xu, R. Halir, Z. Qu, A. Z. Khokhar, A. Osman, W. Cao, C. G. Littlejohns, P. Cheben, I. Molina-Fernández, and G. Z. Mashanovich, Opt. Lett. 43, 795 (2018).

18. W. Zhou, Z. Cheng, X. Wu, B. Zhu, X. Sun, and H. K. Tsang, Opt. Lett. 42, 1245 (2017).

19. D. Chandler-Horowitz and P. M. Amirtharaj, J. Appl. Phys. 97, 123526 (2005).

20. R. Soref, Nat. photonics 4, 495 (2010).

21. M. Nedeljkovic, J. S. Penades, V. Mittal, G. S. Murugan, A. Z. Khokhar, C. Littlejohns, L. G. Carpenter, C. B. E. Gawith, J. S. Wilkinson, and G. Z. Mashanovich, Opt. Express 25, 27431 (2017).

22. D. A. Kozak, T. H. Stievater, R. Mahon, and W. S. Rabinovich, IEEE J. Sel. Top. Quantum Electron. 24, 1 (2018).

23. B. Troia, J. S. Penades, A. Z. Khokhar, M. Nedeljkovic, C. AlonsoRamos, V. M. N. Passaro, and G. Z. Mashanovich, Opt. Lett. 41, 610 (2016).

24. U. Younis, S. K. Vanga, A. E.-J. Lim, P. G.-Q. Lo, A. A. Bettiol, and K.-W. Ang, Opt. Express 24, 11987 (2016).

25. W. Li, P. Anantha, S. Bao, K. H. Lee, X. Guo, T. Hu, L. Zhang, H. Wang, R. Soref, and C. S. Tan, Appl. Phys. Lett. 109, 241101 (2016).

26. J. Kang, M. Takenaka, and S. Takagi, Opt. Express 24, 11855 (2016).

27. J. Kang, X. Yu, M. Takenaka, and S. Takagi, Mater. Sci. Semicond. Process. 42, 259 (2016). E-MRS Spring Meeting 2015 Symposium Z: Nanomaterials and processes for advanced semiconductor CMOS devices.

28. M. Brun, P. Labeye, G. Grand, J.-M. Hartmann, F. Boulila, M. Carras, and S. Nicoletti, Opt. Express 22, 508 (2014).

29. M. A. Ettabib, C. Lacava, Z. Liu, A. Bogris, A. Kapsalis, M. Brun, P. Labeye, S. Nicoletti, D. Syvridis, D. J. Richardson, and P. Petropoulos, Opt. Express 25, 3252 (2017).

30. J. M. Ramirez, Q. Liu, V. Vakarin, J. Frigerio, A. Ballabio, X. L. Roux, D. Bouville, L. Vivien, G. Isella, and D. Marris-Morini, Opt. Express 26, 870 (2018).

31. G. Z. Mashanovich, M. Nedeljkovic, J. Soler-Penades, Z. Qu, W. Cao, A. Osman, Y. Wu, C. J. Stirling, Y. Qi, Y. Cheng, L. Reid, C. G. Littlejohns, J. Kang, Z. Zhao, M. Takenaka, T. Li, Z. Zhou, F. Y. Gardes, D. J. Thomson, and G. T. Reed, Opt. Mater. Express 8, 2276 (2018).

32. J. Kang, Z. Cheng, W. Zhou, T.-H. Xiao, K.-L. Gopalakrisna, M. Takenaka, H. K. Tsang, and K. Goda, Opt. Lett. 42, 2094 (2017).

33. J. R. Jain, D.-S. Ly-Gagnon, K. C. Balram, J. S. White, M. L. Brongersma, D. A. B. Miller, and R. T. Howe, Opt. Mater. Express 1, 1121 (2011).

34. M. M. Krishna and H. H., J. Am. Ceram. Soc. 48, 109.

35. Tydex, "Tydex germanium data sheet," http://www.tydexoptics.com/pdf/ Germanium.pdf.

36. D. E. Hagan and A. P. Knights, J. Opt. 19, 025801 (2017). 